\title{
Merging Cluster Collaboration: Optical and Spectroscopic Survey of a Radio-selected Sample of 29 Merging Galaxy Clusters
}

\author{
N. Golovich ${ }^{1,2}$ (iD), W. A. Dawson ${ }^{1}$ (D), D. M. Wittman ${ }^{2,3}$ (D) M. J. Jee ${ }^{2,4}$, B. Benson ${ }^{2}$ (D), B. C. Lemaux ${ }^{2}$, R. J. van Weeren ${ }^{5,6}$ (D),

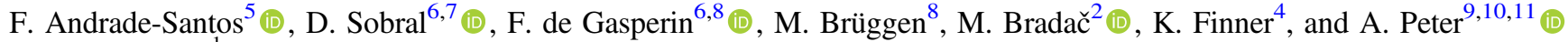 \\ ${ }^{1}$ Lawrence Livermore National Laboratory, 7000 East Avenue, Livermore, CA 94550, USA; golovich1@1lnl.gov \\ ${ }^{2}$ Department of Physics, University of California, One Shields Avenue, Davis, CA 95616, USA \\ ${ }^{3}$ Instituto de Astrofísica e Ciências do Espaço, Universidade de Lisboa, Lisbon, Portugal \\ ${ }^{4}$ Department of Astronomy, Yonsei University, 50 Yonsei-ro, Seodaemun-gu, Seoul, Republic of Korea \\ ${ }^{5}$ Harvard-Smithsonian Center for Astrophysics, 60 Garden Street, Cambridge, MA 02138, USA \\ ${ }^{6}$ Leiden Observatory, Leiden University, P.O. Box 9513, 2300 RA Leiden, The Netherlands \\ 7 Department of Physics, Lancaster University, Lancaster, LA1 4 YB, UK \\ ${ }^{8}$ Hamburger Sternwarte, Universität Hamburg, Gojenbergsweg 112, D-21029 Hamburg, Germany \\ ${ }^{9}$ Department of Astronomy, The Ohio State University, 140 W. 18th Avenue, Columbus, OH 43210, USA \\ ${ }^{10}$ Center for Cosmology and AstroParticle Physics, The Ohio State University, 191 W. Woodruff Avenue, Columbus, OH 43210, USA \\ ${ }^{11}$ Department of Physics, The Ohio State University, $191 \mathrm{~W}$. Woodruff Avenue, Columbus, OH 43210, USA \\ Received 2017 November 3; revised 2018 July 20; accepted 2018 August 3; published 2019 February 13
}

\begin{abstract}
Multi-band photometric and multi-object spectroscopic surveys of merging galaxy clusters allow for the characterization of the distributions of constituent DM and galaxy populations, constraints on the dynamics of the merging subclusters, and an understanding of galaxy evolution of member galaxies. We present deep photometric observations from Subaru/SuprimeCam and a catalog of 4431 spectroscopic galaxies from Keck/DEIMOS observations of 29 merging galaxy clusters ranging in redshift from $z=0.07$ to 0.55 . The ensemble is compiled based on the presence of radio relics, which highlight cluster-scale collisionless shocks in the intracluster medium. Together with the spectroscopic and photometric information, the velocities, timescales, and geometries of the respective merging events may be tightly constrained. In this preliminary analysis, the velocity distributions of 28 of the 29 clusters are shown to be well fit by single Gaussians. This indicates that radio-relic mergers largely occur transverse to the line of sight and/or near-apocenter. In this paper, we present our optical and spectroscopic surveys, preliminary results, and a discussion of the value of radio-relic mergers for developing accurate dynamical models of each system.
\end{abstract}

Key words: galaxies: clusters: general - galaxies: clusters: intracluster medium - galaxies: distances and redshifts large-scale structure of universe

Supporting material: figure set, machine-readable table

\section{Introduction}

Merging galaxy clusters have been established as fruitful astrophysical laboratories. In particular, "dissociative mergers" (Dawson et al. 2012), where two galaxy clusters have collided and the effectively collisionless galaxies and dark matter (DM) have become dissociated from the collisional intracluster medium (ICM), which is disrupted and slows during the merger, are a particularly interesting subclass of mergers. They have been used to place tight constraints on the DM selfinteraction cross section (e.g., Clowe et al. 2006; Randall et al. 2008), to understand fundamental particle/plasma physics associated with the ICM (e.g., Blandford \& Eichler 1987; Markevitch et al. 2002; Brunetti \& Jones 2014; van Weeren et al. 2017), and merger related galaxy evolution (e.g., Miller \& Owen 2003; Poggianti et al. 2004; Chung et al. 2009; Stroe et al. 2014, 2017; Mansheim et al. 2017a, 2017b). These studies have allowed for a new and broader understanding of the content, distribution, and interactions between and within each component. However, they are complicated by: the complexity of the merger properties (mass, dynamics, etc.), the range of disparate observations necessary to form a synoptic understanding of any one merger, and the limited sample size of dissociative mergers to study.
Mergers are complex physical phenomena in which dynamical parameters such as the merger speed at pericenter, the elapsed time since pericenter, and the merger geometry are typically unknown. This leaves a vast volume of parameter space that must be considered in any subsequent analysis to properly propagate uncertainty (e.g., Lage \& Farrar 2015). The volume of parameter space that must be explored can be shrunk by studying the separate components of the merger as a whole (e.g., Dawson 2013; Ng et al. 2015; Golovich et al. 2016).

Observationally, each component of a merger is probed differently. The DM must be inferred using gravitational lensing techniques that necessitate deep photometric images (see Bartelmann \& Schneider 2001; Hoekstra 2013, for a review). The ICM is hot ( $\sim$ several $\mathrm{keV})$ and emits thermal bremsstrahlung X-rays (e.g., Cavaliere \& Fusco-Femiano 1976), which may be observed spatially and spectroscopically with modern X-ray observatories in orbit. Non-thermal emission from the ICM may be observed with radio telescopes, which reveal complex microphysics of particle acceleration and turbulence (see, e.g., Brunetti et al. 2008). The galaxies may be observed photometrically and spectroscopically. Photometry in multiple bands allow for semi-precise photometric redshift estimates (see, e.g., Benítez 2000; Bolzonella et al. 2000) 
and red sequence selection of cluster members (e.g., Kodama \& Arimoto 1997). Spectroscopic observations, in contrast, allow for precise redshift estimation, but these observations are much more expensive and usually result in incomplete surveys of member galaxies. Spectroscopy may also be used to study the effects of the cluster environment on the constituent galaxies via line ratios (e.g., Baldwin et al. 1981), including AGN and star formation rate studies (e.g., Moore et al. 1996; Miller \& Owen 2003; Stroe et al. 2014; Sobral et al. 2015).

Circa 2012, all dissociative mergers were identified and confirmed using an array of aforementioned observations. Collecting and analyzing this array of observations was resource-intensive, which in large part is the reason for the small sample of dissociative mergers (see Dawson et al. 2012 for a list of the eight known dissociative mergers in 2012). In recent years, we have implemented new techniques of quickly identifying dissociative merging galaxy clusters via detection of enhanced, diffuse radio emission. Radio relics and radio halos appear in radio images between $\sim 100 \mathrm{MHz}$ and several $\mathrm{GHz}$ as megaparsec-scale, diffuse radio features. They are thought to trace synchrotron emission from electrons interacting with shocks and turbulent motion (e.g., Brunetti et al. 2008; Feretti et al. 2012), and thus should be associated with dissociative mergers. Magnetohydrodynamical simulations of cluster mergers confirm this, and can reporduce key features of radio relics (e.g., Skillman et al. 2013; Vazza et al. 2016). Because radio-relic selection of dissociative mergers can be done with a single-band wide-field survey, while maintaining a high purity (as demonstrated in this paper), it is more economical compared to previous multi-probe selection methods.

Spectroscopic and photometric observations of the galaxies of merging subclusters allow for estimation of the dynamical properties of individual merging systems. We have demonstrated this with a series of studies of individual merger systems (CIZA J2242.8+5301, El Gordo, MACS J1149.5+2223, ZwCl 0008.8+5215, A3411, and $\mathrm{ZwCl} 2341.1+0000$ presented in Dawson et al. 2015; Ng et al. 2015; Golovich et al. 2016, 2017; Benson et al. 2017; van Weeren et al. 2017, respectively). The dynamical models of individual clusters greatly reduce the vast parameter space that simulators must explore to reproduce underlying astrophysics. The presence of radio relics in each of these systems has been shown to greatly improve the precision of dynamical models ( $\mathrm{Ng}$ et al. 2015; Golovich et al. 2016, 2017), and direct study of the underlying shock and radio relics has yielded insight into particle acceleration models (e.g., Brunetti \& Jones 2014; van Weeren et al. 2017).

In this paper, we outline our photometric and spectroscopic observations of an ensemble of 29 radio-relic mergers. In Section 2 we describe the construction of the ensemble of 29 merging systems. In Section 3 we detail our photometric and spectroscopic observational campaign, including the technical details of the observations, data reduction, and data processing. We compile and analyze the redshift global redshift distributions of each system in Section 4, and we discuss the implications of radio selection and offer conclusions in Section 5.

We assume a flat $\Lambda \mathrm{CDM}$ universe with $H_{0}=$ $70 \mathrm{~km} \mathrm{~s}^{-1} \mathrm{Mpc}^{-1}, \Omega_{M}=0.3$, and $\Omega_{\Lambda}=0.7$. AB magnitudes are utilized throughout, and all distances are proper.

\section{Radio-relic Sample}

Constraining the DM self-interaction cross section is one of the driving science cases for this survey. A radio-relic selection has a number of potential advantages for this science case over other selection methods: (1) it guarantees against the selection of pre-pericentric systems because the presence of a radio-relic indicates a shockwave traveling in the ICM due to a major merger; (2) it will disfavor the very youngest post-pericentric systems, which have not had time to generate radio relics, and where the offset between the effectively collisionless galaxies and potentially self-interacting DM has not had a chance to increase to a potential maximal offset (Kahlhoefer et al. 2014); (3) it is biased toward selecting mergers in the plane of the sky where any observable offset between the galaxies, DM, and ICM will be maximized (this is also important for other astrophysical studies; Ensslin et al. 1998); and (4) as noted in Section 1, a large sample of dissociative mergers can be prudently compiled.

The first detection of a radio relic in a merging galaxy cluster was in the Coma Cluster (Ballarati et al. 1981). Radio relics were subsequently discovered individually through pointed observations of known merging systems. In the last decade, searches of wide-area radio surveys have increased the rate of detection (e.g., van Weeren et al. 2011a). Several potential radio relics were discovered through comparisons of radio surveys with the ROSAT All-Sky Survey catalogs (Voges et al. 1999). Follow-up of these objects resulted in several discoveries (van Weeren et al. 2009a, 2009b, 2010, 2011b, 2012a, 2012b, 2013). Our sample begins with these radio relics, along with additional radio relics known by 2011 September listed in Table 3 of Feretti et al. (2012). Each of these clusters contains low-surface-brightness, steep-spectrum, polarized, and extended radio sources that lie at the periphery of the cluster (for individual observational papers see references therein). Relics classified as having a round morphology were discarded because they are likely radio phoenixes rather than megaparsec-scale cluster shocks. Radio phoenixes are generally associated with aged radio galaxy lobes that are re-energized through compression or other mechanisms (e.g., de Gasperin et al. 2015b). We imposed cuts designed to enable spectroscopic and weak lensing follow-up. Systems at very low redshift are not efficient lenses, so we eliminate clusters at $z<0.07$. We also eliminate systems not observable from the Maunakea observatories $\left(\delta<-31^{\circ}\right)$ from which we were awarded observational time. To this list, we added three additional radio-relic systems that have appeared in the literature and pass the same selection criteria (MACS J1149.5+2223, PSZ1 G108.18-11.5 and ZwCl 1856+6616, hereafter MACSJ1149, PSZ1G108 and ZwC11856, respectively: Bonafede et al. 2012; de Gasperin et al. 2014, 2015a). Finally, we added one of the radio phoenix relics (Abell 2443, hereafter A2443) to our spectroscopic survey due to a gap in an observing run. In total, our sample contains 29 systems; they are listed in Table 1.

The sample is predominantly composed of low-redshift $(\sim 0.1-0.3)$ clusters due to radio relics typically being discovered in wide, shallow surveys (e.g., NVSS: Condon et al. 1998). This is a reasonable redshift range for lensing follow-up, and also has the advantage of mapping given physical separations to substantial angular separations for spatial analysis of cluster components. 
Table 1

The Merging Cluster Collaboration Radio-selected Sample

\begin{tabular}{|c|c|c|c|c|c|}
\hline Cluster & Short name & R.A. & Decl. & Redshift & Discovery Band \\
\hline 1RXS J0603.3+4212 & 1RXSJ0603 & 06:03:13.4 & $+42: 12: 31$ & 0.226 & Radio \\
\hline Abell 115 & A115 & $00: 55: 59.5$ & $+26: 19: 14$ & 0.193 & Optical \\
\hline Abell 521 & A521 & 04:54:08.6 & $-10: 14: 39$ & 0.247 & Optical \\
\hline Abell 746 & A746 & 09:09:37.0 & $+51: 32: 48$ & 0.214 & Optical \\
\hline Abell 781 & A781 & $09: 20: 23.2$ & $+30: 26: 15$ & 0.297 & Optical \\
\hline Abell 1240 & A1240 & $11: 23: 31.9$ & $+43: 06: 29$ & 0.195 & Optical \\
\hline Abell 2034 & A2034 & $15: 10: 10.8$ & $+33: 30: 22$ & 0.114 & Optical \\
\hline Abell 2061 & A2061 & $15: 21: 20.6$ & $+30: 40: 15$ & 0.078 & Optical \\
\hline Abell 2163 & A2163 & $16: 15: 34.1$ & $-06: 07: 26$ & 0.201 & Optical \\
\hline Abell 2255 & A2255 & $17: 12: 50.0$ & $+64: 03: 11$ & 0.080 & Optical \\
\hline Abell 2345 & A2345 & $21: 27: 09.8$ & $-12: 09: 59$ & 0.179 & Optical \\
\hline Abell 2443 & A2443 & $22: 26: 02.6$ & $+17: 22: 41$ & 0.110 & Optical \\
\hline MACS J1149.5+2223 & MACSJ1149 & $11: 49: 35.8$ & $+22: 23: 55$ & 0.544 & X-ray \\
\hline MACS J1752.0+4440 & MACSJ1752 & $17: 52: 01.6$ & $+44: 40: 46$ & 0.365 & X-ray \\
\hline PLCKESZ G287.0+32.9 & PLCKG287 & $11: 50: 49.2$ & $-28: 04: 37$ & 0.383 & $\mathrm{SZ}$ \\
\hline PSZ1 G108.18-11.53 & PSZ1G108 & $23: 22: 29.7$ & $+48: 46: 30$ & 0.335 & $\mathrm{SZ}$ \\
\hline RXC J1053.7+5452 & RXCJ1053 & $10: 53: 44.4$ & $+54: 52: 21$ & 0.072 & X-ray \\
\hline RXC J1314.4-2515 & RXCJ1314 & $13: 14: 23.7$ & $-25: 15: 21$ & 0.247 & $\mathrm{X}$-ray \\
\hline $\mathrm{ZwCl} 0008.8+5215$ & $\mathrm{ZwCl0008}$ & $00: 08: 25.6$ & $+52: 31: 41$ & 0.104 & Optical \\
\hline $\mathrm{ZwCl} 1447+2619$ & ZwCl1447 & $14: 49: 28.2$ & $+26: 07: 57$ & 0.376 & Optical \\
\hline $\mathrm{ZwCl} 1856.8+6616$ & ZwCl1856 & $18: 56: 41.3$ & $+66: 21: 56$ & 0.304 & Optical \\
\hline $\mathrm{ZwCl} 2341+0000$ & $\mathrm{ZwCl} 2341$ & $23: 43: 39.7$ & $+00: 16: 39$ & 0.270 & Optical \\
\hline
\end{tabular}

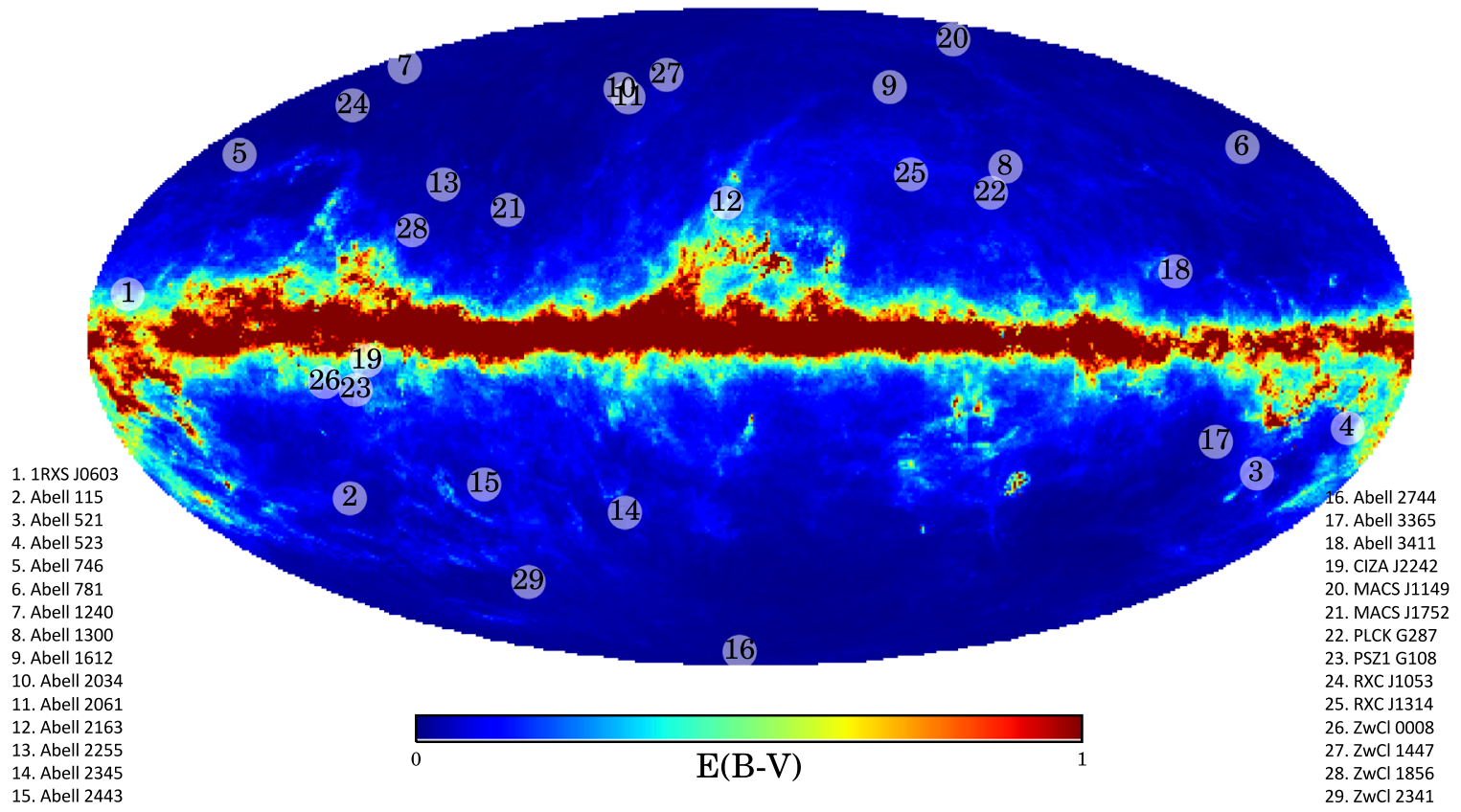

Figure 1. Galactic dust extinction map (Schlegel et al. 1998) with the overlaid positions of the 29 systems in our sample.

The radio selection strategy brings challenges in terms of obtaining spectroscopy and lensing follow-up. Because radio surveys have gone right through the galactic plane, many of the systems suffer more extinction than is typical in visiblewavelength surveys. The all-sky galactic dust extinction map is presented in Figure 1 with all 29 systems in our sample. The most extreme example is CIZAJ2242 with $A_{V} \approx 1.4$ (the approximation sign emphasizes that the extinction varies over the field; Schlegel et al. 1998). Dawson et al. (2015) described the success of the position-dependent extinction corrections applied to that system in terms of yielding uniform color selection of cluster members, and Jee et al. (2015) 
demonstrated that weak lensing can be efficiently measured despite the extinction. The low galactic latitude also affected the spectroscopy not only through extinction but also by causing more slits to be wasted on stars. A contributing factor in some cases was the poor quality of imaging available at the time of slit-mask design. Blended binary stars were not rejected in morphological cuts, and constituted a substantial contamination. The next most extinct systems in the sample are A2163

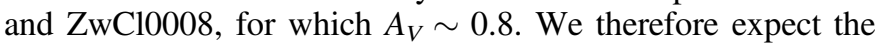
lensing and galaxy analyses of most of the systems in this sample to exceed the quality of those for CIZAJ2242. We have corrected our photometry for extinction throughout.

The resulting 29 systems are listed in Table 1. For each system, the following milestones are to be achieved for each cluster:

1. Observations including spectroscopic, ground-based wide-field photometric, space-based pointed photometric, X-ray, and radio;

2. Optical analysis to estimate the number and location of subclusters;

3. Redshift analysis to estimate the line-of-sight velocity information of subclusters;

4. X-ray and radio analysis of shocks and radio relics, including polarization measurements;

5. Weak-lensing analysis to find the location and mass of subclusters; and

6. Dynamical analysis.

In this paper, we will discuss the spectroscopic and widefield optical observations for our sample of 29 merging clusters. These will ultimately result in two of the three primary inputs for the dynamics analysis as well as classify the mergers by their complexity and reasonability to probe astrophysical hypotheses including merging-induced galaxy evolution, particle acceleration at cluster shocks, mergerinduced turbulence, and self-interacting DM models. The remaining goals will be achieved in follow-up papers utilizing the data presented here.

\section{Optical Imaging and Spectroscopic Observational Campaign}

\subsection{Survey Goals and Requirements}

The goal of the optical imaging survey is to obtain lensingquality, wide-field imaging in at least two photometric bands. The two filters are chosen to straddle the $4000 \AA$ break in order to select cluster members photometrically via red sequence relations. Furthermore, our weak lensing method makes use of these red sequence relations in order to select background galaxies for lensing studies (Jee et al. 2015, 2016; Golovich et al. 2017). Additionally, for clusters for whom our SuprimeCam observations came before our DEIMOS observations, we made use of the SuprimeCam images for spectroscopic target selection (see Section 3.3.1). Many clusters have archival imaging that we have obtained. We observed 18 systems with Subaru/SuprimeCam to complete the photometric survey.

The spectroscopic survey has a goal of obtaining $\sim 200$ member galaxy velocities in each system. We used redshifts from the literature when available in order to reduce the amount of new observations required. When obtaining new spectra, we designed observations to also meet the goal of enabling studies of recent star formation and ultimately the link between mergers and star formation. We achieve this by adjusting the observed wavelength range for each cluster to the emitted wavelength range from $\mathrm{H} \beta$ to $\mathrm{H} \alpha$ for clusters with $z \lesssim 0.3$ and [O II] to [O III] for clusters with $z \gtrsim 0.3$. The data available for star formation studies therefore varies from cluster to cluster, depending on the number of previously published redshifts and the redshift of the cluster. Additional observations were required for 18 systems, with many having no more than a handful of previously published member redshifts. In the following subsections we will detail the targeting, observing, and data reductions of our optical and spectroscopic surveys.

\subsection{Subaru/SuprimeCam Observations}

We observed 18 clusters over four nights using the 80 Megapixel SuprimeCam (Miyazaki et al. 2002) camera on the Subaru Telescope on Maunakea. Table 2 summarizes these observations. The basic strategy is to achieve weak-lensing quality in one filter and obtain a second filter to define the color of detected objects by straddling the $4000 \AA$ break. For the lensing-quality image, the exposure time was $2880 \mathrm{~s}(8 \times 360$ s) and we rotated the field between each exposure by $15^{\circ}$ in order to distribute the bleeding trails and diffraction spikes from bright stars azimuthally to be later removed by medianstacking. This scheme enabled us to maximize the number of detected galaxies, especially for background source galaxies for weak lensing near stellar halos or diffraction spikes. In the second and third filters ( $g$ and/or $i$ ), the exposure time was $720 \mathrm{~s}(4 \times 180 \mathrm{~s})$. These exposures were rotated by $30^{\circ}$ from exposure to exposure for the same reason as above. In order to efficiently fill the time of each observing night, we added a third band to several clusters. The actual observing times may vary due to real-time changes to the observational plan due to unexpected lost time.

Archival Subaru/SuprimeCam imaging was downloaded from the SMOKA data archive (Baba et al. 2002), and is detailed in Table 3. We note that the observational strategy for the archival data did not prescribe rotating between exposures, so diffraction spikes and bleeding trails are present. Also, we did not make use of the full set of archival images for these clusters because we only required two bands of imaging in order to define the color and complete a color-magnitude selection. We utilized the deepest images available that satisfy this requirement, ensuring good seeing conditions.

\subsubsection{Subaru/SuprimeCam: Data Reduction}

The CCD processing (overscan subtraction, flat-fielding, bias correction, initial geometric distortion rectification, etc) was carried out with the SDFRED2 package (Ouchi et al. 2004). Much of the archival data required the first version of this pipeline (SDFRED1: Yagi et al. 2002). We refine the geometric distortion and World Coordinate System information using the SCAMP software (Bertin 2006). The Two Micron All Sky Survey (2MASS; Skrutskie et al. 2006) catalog was selected as a reference when the SCAMP software was run except for clusters covered by the Sloan Digital Sky Survey (SDSS; Adelman-McCarthy et al. 2007), for which the Data Release 5 catalogs were used. We also rely on SCAMP to calibrate out the sensitivity variations across different frames. For image stacking, we ran the SWARP software (Bertin et al. 2002) using the SCAMP result as input. We first created median 
Table 2

Merging Cluster Collaboration Radio-relic-selected Subaru/SuprimeCam Survey

\begin{tabular}{|c|c|c|c|c|}
\hline Cluster & Filter & Date & Seeing $(\operatorname{arcsec})$ & Exposure (s) \\
\hline 1RXS J060313.4+421231 & $g$ & 2014 Feb 25 & 0.57 & 720 \\
\hline 1RXS J060313.4+421231 & $r$ & 2014 Feb 25 & 0.57 & 2880 \\
\hline 1RXS J060313.4+421231 & $i$ & 2014 Feb 25 & 0.50 & 720 \\
\hline Abell 523 & $g$ & 2014 Feb 26 & 1.00 & 720 \\
\hline Abell 523 & $r$ & 2014 Feb 26 & 0.78 & 2880 \\
\hline Abell 746 & $g$ & 2014 Feb 26 & 0.88 & 720 \\
\hline Abell 746 & $r$ & 2014 Feb 26 & 1.01 & 2880 \\
\hline Abell 1240 & $g$ & 2014 Feb 25 & 0.67 & 720 \\
\hline Abell 1240 & $r$ & 2014 Feb 25 & 0.58 & 2880 \\
\hline Abell 1300 & $g$ & 2014 Feb 26 & 0.89 & 720 \\
\hline Abell 1300 & $r$ & 2014 Feb 26 & 0.88 & 2160 \\
\hline Abell 1612 & $g$ & 2014 Feb 25 & 0.62 & 720 \\
\hline Abell 2061 & $g$ & 2013 Jul 13 & 0.68 & 720 \\
\hline Abell 2061 & $r$ & 2013 Jul 13 & 0.67 & 2520 \\
\hline Abell 2061 & $i$ & 2013 Jul 13 & 0.60 & 2676 \\
\hline Abell 2061 & $i$ & 2014 Feb 26 & 0.65 & 720 \\
\hline Abell 3365 & $g$ & 2014 Feb 25 & 0.97 & 720 \\
\hline Abell 3365 & $r$ & 2014 Feb 25 & 0.71 & 2880 \\
\hline Abell 3365 & $i$ & 2014 Feb 25 & 0.62 & 720 \\
\hline Abell 3411 & $g$ & 2014 Feb 25 & 0.80 & 720 \\
\hline Abell 3411 & $r$ & 2014 Feb 25 & 0.82 & 2880 \\
\hline Abell 3411 & $i$ & 2014 Feb 25 & 0.77 & 720 \\
\hline CIZA J2242.8+5301 & $g$ & 2013 Jul 13 & 0.63 & 720 \\
\hline CIZA J2242.8+5301 & $i$ & $2013 \mathrm{Jul} 13$ & 0.55 & 3400 \\
\hline MACS J175201.5+444046 & $g$ & $2013 \mathrm{Jul} 13$ & 0.62 & 720 \\
\hline MACS J175201.5+444046 & $r$ & 2013 Jul 13 & 0.64 & 1440 \\
\hline MACS J175201.5+444046 & $i$ & 2013 Jul 13 & 0.63 & 2520 \\
\hline MACS J175201.5+444046 & $i$ & 2014 Feb 26 & 0.73 & 1260 \\
\hline PLCKESZ G287.0+32.9 & $g$ & 2014 Feb 26 & 0.81 & 720 \\
\hline PLCKESZ G287.0+32.9 & $r$ & 2014 Feb 26 & 0.97 & 2880 \\
\hline PSZ1 G108.18-11.53 & $g$ & 2015 Sep 12 & 0.65 & 1440 \\
\hline PSZ1 G108.18-11.53 & $r$ & 2015 Sep 12 & 0.55 & 2520 \\
\hline RXC J1053.7+5452 & $g$ & 2014 Feb 26 & 0.83 & 720 \\
\hline RXC J1053.7+5452 & $r$ & 2014 Feb 26 & 0.92 & 720 \\
\hline RXC J1314.4-2515 & $g$ & 2014 Feb 25 & 0.86 & 720 \\
\hline RXC J1314.4-2515 & $r$ & 2014 Feb 25 & 0.71 & 2880 \\
\hline RXC J1314.4-2515 & NB814 & 2014 Feb 25 & 0.77 & 1000 \\
\hline $\mathrm{ZwCl} 0008.8+5215$ & $g$ & 2013 Jul 13 & 0.52 & 720 \\
\hline $\mathrm{ZwCl} 0008.8+5215$ & $r$ & $2013 \mathrm{Jul} 13$ & 0.57 & 2880 \\
\hline $\mathrm{ZwCl} 1447+2619$ & $g$ & 2014 Feb 26 & 0.91 & 720 \\
\hline $\mathrm{ZwCl} 1447+2619$ & $r$ & 2014 Feb 26 & 0.76 & 2880 \\
\hline $\mathrm{ZwCl} 1447+2619$ & $i$ & 2014 Feb 26 & 0.55 & 720 \\
\hline $\mathrm{ZwCl} 1856+6616$ & $g$ & 2015 Sep 12 & 0.70 & 720 \\
\hline $\mathrm{ZwCl} 1856+6616$ & $r$ & 2015 Sep 12 & 0.65 & 2520 \\
\hline $\mathrm{ZwCl} 2341+0000$ & $g$ & 2013 Jul 13 & 0.49 & 720 \\
\hline $\mathrm{ZwCl} 2341+0000$ & $r$ & $2013 \mathrm{Jul} 13$ & 0.50 & 2880 \\
\hline
\end{tabular}

mosaic images and then used it to mask out pixels ( $3 \sigma$ outliers) in individual frames. These masked frames were weightaveraged to generate the final mosaic, which is used for the scientific analyses hereafter. Two example images are presented in Figure 2.

\subsubsection{Subaru/SuprimeCam: Photometric Catalog Generation}

Object detection is achieved with Source Extractor (Bertin \& Arnouts 1996) in dual image mode using the deepest image for detection. The blending threshold parameter BLENDNTHRESH is set to 32 with a minimal contact DEBLEND_MINCONT of $10^{-4}$. We employ reddening values from Schlafly \& Finkbeiner (2011) to correct for dust extinction, which are listed in Tables 2 and 3. Zero-points were transferred from SDSS for the overlapping clusters and transferred to the clusters outside the SDSS footprint observed on the same night with SuprimeCam accounting for atmospheric extinction related to the airmass differences of our observations. Atmospheric extinction values for Maunakea were taken from Buton et al. (2013). Zero-points for Subaru observations with filters outside the SDSS ugriz filter set were computed following Jester et al. (2005). These included the $V$ band for A115 and the $R$ band for A2034. Both clusters are in the SDSS footprint.

Since the sample has relatively low redshift, it is expected for cluster members to have high signal-to-noise $(\mathrm{S} / \mathrm{N})$ and correspondingly good photometry. We enforce that potential cluster member objects have uncertainties in their magnitudes of less than $0.5 \mathrm{mag}$, and we remove all objects brighter than the BCG, which we have identified spectroscopically in each cluster. These cuts eliminate most bright-foreground galaxies 

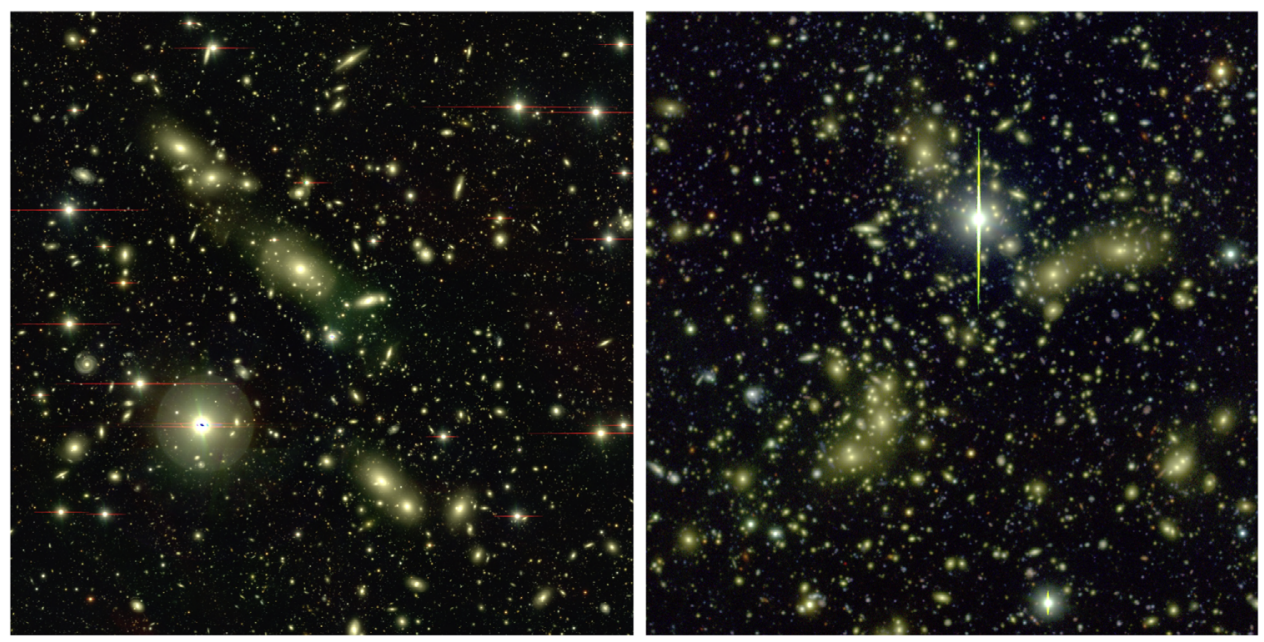

Figure 2. Example Subaru/SuprimeCam images of the central regions of A2061 (left) and A2744 (right). A2061 is displayed using our $g$, $r$, and $i$ images while A2744 is displayed using archival $B, R$, and $Z$ images. Note that the $i$-band image for A2061 and the $Z$-band image for A2744 were used only to make these true-color images. These images were combined using the trilogy software (Coe et al. 2012).

Table 3

Archival Imaging from Subaru/SuprimeCam Utilized in This Study

\begin{tabular}{lllc}
\hline \hline Cluster & Filter & Date & Exposure (s) \\
\hline Abell 115 & $V$ & 2003 Sep 25, 2005 October 03 & 1530 \\
Abell 115 & $i$ & 2005 Oct 3 & 2100 \\
Abell 521 & $V$ & 2001 Oct 14 & 1800 \\
Abell 521 & $R$ & 2001 Oct 15 & 1620 \\
Abell 781 & $V$ & 2010 Mar 14, 15 & 3360 \\
Abell 781 & $i$ & 2010 Mar 15 & 2160 \\
Abell 1612 & $i$ & 2010 Apr 11 & 1920 \\
Abell 2034 & $g$ & 2005 Apr 11 & 720 \\
Abell 2034 & $R$ & 2005 Apr 11, 2007 June 19 & 12880 \\
Abell 2163 & $V$ & 2009 Apr 30 & 2100 \\
Abell 2163 & $R$ & 2008 Apr 7 & 4500 \\
Abell 2255 & $B$ & 2007 Aug 14 & 1260 \\
Abell 2255 & $R$ & 2007 Aug 14 & 2520 \\
Abell 2345 & $V$ & 2010 Jun 10, 2010 Nov 10 & 3600 \\
Abell 2345 & $i$ & 2005 Oct 3 & 2100 \\
Abell 2744 & $B$ & 2013 Jul 16 & 2100 \\
Abell 2744 & $R$ & 2013 Jul 15 & 3120 \\
MACS J1149 & $V$ & 2003 Apr 5 & 2520 \\
MACS J1149 & $R$ & 2003 Apr 5, 2005 Mar 5, 2010 & 5490 \\
& & Mar 18 & \\
\hline
\end{tabular}

and stars, as well as false detections at extremely faint magnitudes. Only objects within $R_{200}$ (as determined from our redshift analysis and scaling relations Duffy et al. 2008; Evrard et al. 2008) of the center of the cluster are retained. $R_{200}$ is a common measure of cluster radius and is defined such that the sphere of radius $R_{200}$ has a mean density $\bar{\rho}=200 \rho_{c}$, where $\rho_{c}$ is the critical density of the universe. This cut limits the vignetting of the edges as well as removes spurious detections near the edge of the field.

\subsection{Keck/DEIMOS Observations}

We conducted a spectroscopic survey utilizing the DEIMOS multi-object spectrograph (Faber et al. 2003) on the Keck II telescope at the W. M. Keck Observatory on Maunakea over the following nights: 2013 January 26, 2014 July 14, 2014 September 5, 2013 December 3-5 (half nights), 2014 June 22-23, 2015 February 15 , and 2015 December 13. In total, 54 slit-masks were observed. Each was milled with $1^{\prime \prime}$ wide slits and utilized the 1200 line $\mathrm{mm}^{-1}$ grating, which results in a pixel scale of $0.33 \AA$ pixel $^{-1}$ and a resolution of $\sim 1 \AA\left(50 \mathrm{~km} \mathrm{~s}^{-1}\right)$. For clusters with a redshift below 0.3 , the grating was tilted to observe the following spectral features: $\mathrm{H} \beta,[\mathrm{O} \mathrm{III}], \mathrm{Mg}$ I (b), Fe I, NaI (D), [O I], $\mathrm{H} \alpha$, and the [N II] doublet. A typical wavelength coverage of 5400 to $8000 \AA$ is shown in Figure 3 for a galaxy observed in CIZAJ2242. The actual wavelength coverage may be shifted by $\sim \pm 400 \AA$ depending where the slit is located along the width of the slit-mask. This spectral setup enables us to also study the star formation properties of the cluster galaxies; see related work by Sobral et al. (2015). For higher-redshift clusters (above 0.3), the grating was tilted to instead cover the following spectra features: [O II], $\mathrm{Ca}(\mathrm{H})$, $\mathrm{Ca}(\mathrm{K}), \mathrm{H} \delta, G$-band, $\mathrm{H} \gamma, \mathrm{H} \beta$, and [O III]. The position angle (PA) of each slit was chosen to lie between $\pm 5^{\circ}$ and $30^{\circ}$ of the slitmask PA to achieve optimal sky subtraction during reduction with the DEEP2 version of the spec2d package (Newman et al. $2013 \mathrm{~b}$ ). In general, for each mask we took three $\sim 900 \mathrm{~s}$ exposures except for a few cases where a few extra minutes at the end of the night were spent on an individual mask or when weather altered our observation plans in the middle of the night. In total, 54 slit-masks were observed with a total of $\sim 7000$ slits over the course of the spectroscopic survey.

\subsubsection{Keck/DEIMOS: Target Selection}

Our primary objective for the spectroscopic survey was to maximize the number of cluster member spectroscopic redshifts in order to detect merging substructure. For each slitmask, the best imaging data available were utilized. For onethird of the clusters this was our own SuprimeCam imaging from our simultaneous wide-field imaging survey (see Section 3.2). In the cases where this was unavailable at the time of our spectroscopic survey planning, we used the next best imaging at our disposal. SDSS Data Release 5 catalogs were utilized (Adelman-McCarthy et al. 2007) for 10 of the clusters, and for 6 of the clusters, this was INT WFC data presented in van Weeren et al. (2011c). For the remaining two clusters, Digitized Sky Survey (Djorgovski et al. 1992) imaging was utilized. For all imaging except the SDSS data, for which a photometric redshift selection was employed, a red sequence 


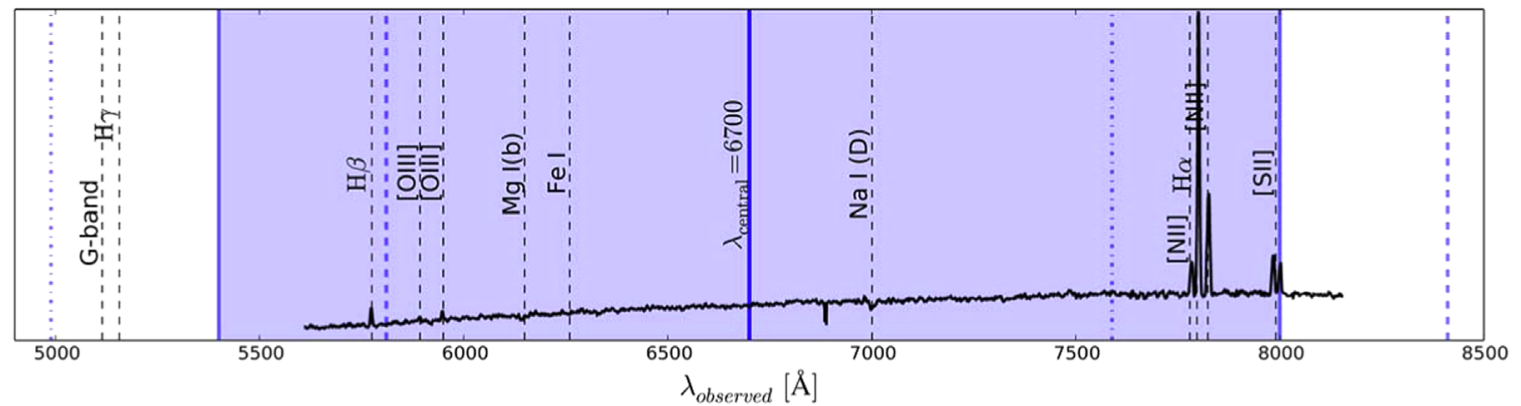

Figure 3. Reprinted Figure 2 of Dawson et al. (2015). Example spectral coverage of the Keck/DEIMOS observations (shaded blue region) for a low-redshift $(z \leqslant 0.3$ ) cluster, along with the redshifted location of common cluster emission and absorption features (black dashed lines). The blue dotted-dashed pair and the blue dashed pair of lines show the variable range depending on where the slit was located along the width of the slit-mask. The solid black line shows an example galaxy spectrum from our DEIMOS survey.

technique was utilized to select likely cluster members to create a galaxy number density map. The slit-masks were then oriented to maximize the number of cluster members in the high red sequence density regions. Priors from the literature were also utilized in the placement of slit-masks (e.g., lensing maps, X-ray surface brightness, radio relics, etc).

The DEIMOS $5^{\prime} \times 16^{\prime}$.7 field of view is very well suited to survey the low-z, elongated merging systems in our sample. In most cases, we aligned the long axis of our slit-masks with the long axis of the system. The success of star-galaxy separation in our targeting data was variable and depended on the seeing of the imaging; thus, several of our slit-masks were highly contaminated with stars. For example, CIZAJ2242, which sits near the plane of the galaxy, has a stellar density nearly three times that of cluster members. When selecting targets, we divided our potential targets into a bright red sequence sample (Sample 1; $r<22.5$ ) and a faint red sequence sample (Sample $2 ; 22.5<r<23.5)$. We first filled our mask with as many Sample 1 targets as possible, then filled in the remainder of the mask with Sample 2 targets. While we preferentially targeted likely red sequence cluster members it was not always possible to fill the entire mask with these galaxies, in which case we would place a slit on bright blue cloud galaxies in the field. For the SDSS-targeted galaxies, we selected from galaxies satisfying $z_{\text {phot }}$ within $\pm 0.05\left(1+z_{\text {cluster }}\right)$ of the cluster redshift and prioritized bright galaxies with a luminosity-weighted selection. In these cases, Sample 2 was composed of any other bright objects outside the photometric selection.

We used the DSIMULATOR package ${ }^{12}$ to design each slitmask. DSIMULATOR automatically selects targets by maximizing the sum total weights of target candidates, by first selecting as many objects from Sample 1 as possible then filling in the remaining area of the slit-mask with target candidates from Sample 2. We manually edited the automated target selection to increase the number of selected targets, e.g., by selecting another target between targets selected automatically by DSIMULATOR if the loss of sky coverage was acceptably small.

In Table 4, we summarize the survey design aspects for all 54 of our slit-masks.

\subsubsection{Keck/DEIMOS: Data Reduction}

The exposures for each mask were combined using the DEEP2 versions of the spec $2 d$ and spec1d packages (Newman

\footnotetext{
12 http://www.ucolick.org/ phillips/deimos_ref/masks.html
}

et al. 2013a). This package combines the individual exposures of the slit mosaic and performs wavelength calibration, cosmic ray removal and sky subtraction on a slit-by-slit basis, generating a processed two-dimensional spectrum for each slit. The spec $2 d$ pipeline also generates a processed one-dimensional spectrum for each slit. This extraction creates a one-dimensional spectrum of the target, containing the summed flux at each wavelength in an optimized window. The spec1d pipeline then fits template spectral energy distributions (SEDs) to each one-dimensional spectrum and estimates a corresponding redshift. There are SED templates for various types of stars, galaxies, and active galactic nuclei. We then visually inspect the fits using the zspec software package (Newman et al. 2013b), assign quality rankings to each fit (following a convention closely related to Newman et al. 2013b), and manually fit for redshifts where the automated pipeline failed to identify the correct fit. The highest quality galaxy spectra $(\mathrm{Q}=4)$ have a mean $\mathrm{S} / \mathrm{N}$ of 10.7 per pixel, while the minimum quality galaxy spectra used on our redshift analysis $(Q=3)$ have a mean $\mathrm{S} / \mathrm{N}$ of 4.9 per pixel. Note that the $\mathrm{S} / \mathrm{N}$ estimates are dominated by the continuum of a spectroscopic trace and an emission line galaxy may be of high quality but very low mean $\mathrm{S} / \mathrm{N}$ (for example the mean $\mathrm{S} / \mathrm{N}$ of a $Q=4$ emission line galaxy is 1.2 despite detection of $\mathrm{H} \alpha$ and $\mathrm{H} \beta$ or [O III] in most cases). An example of one of the reduced spectra is reprinted from Figure 2 of Dawson et al. (2015) in Figure 3 and more are shown in a related galaxy evolution paper (Sobral et al. 2015).

In Table 7, we present 4340 high-quality galaxies (including foreground and background to the cluster) from our spectroscopic survey, along with matched photometry from our photometric survey.

\subsubsection{Archival Spectroscopy}

To augment our spectroscopic survey, we completed a detailed literature review of published spectroscopic redshifts of cluster members for the 29 systems in the ensemble. We compiled spectroscopic galaxies in each field using the NASA/ IPAC Extragalactic Database ${ }^{13}$ (NED). For each system we considered galaxies within $5 \mathrm{Mpc}$ of the cluster center and within $\pm 10,000 \mathrm{~km} \mathrm{~s}^{-1}$ of the mean cluster redshift to be sufficiently plausible members. Many galaxies published in the literature also appear in NED, so we cross matched and eliminated duplicate galaxies and prioritized originally published galaxies over NED matches.

\footnotetext{
13 http://ned.ipac.caltech.edu/
} 
Table 4

Merging Cluster Collaboration Radio-relic-selected Spectroscopic Survey

\begin{tabular}{|c|c|c|c|c|c|}
\hline Slit-mask & Date & Target Imaging & Exposure (s) & Wavelength $(\AA)$ & Slits \\
\hline 1RXSJ0603-1 & 2013 Jan 16 & WFC & 3000 & 6200 & 105 \\
\hline 1RXSJ0603-2 & 2013 Jan 16 & WFC & 3000 & 6200 & 100 \\
\hline 1RXSJ0603-3 & 2013 Sep 5 & WFC & 3600 & 7000 & 98 \\
\hline 1RXSJ0603-4 & 2013 Sep 5 & WFC & 3600 & 7000 & 87 \\
\hline A115-1 & 2014 Jun 22 & SDSS & 2500 & 6900 & 176 \\
\hline A115-2 & 2014 Jun 23 & SDSS & 2400 & 6900 & 142 \\
\hline A523-1 & 2013 Jan 16 & WFC & 3000 & 6200 & 99 \\
\hline A523-2 & 2013 Dec 4 & WFC & 2700 & 6200 & 94 \\
\hline A523-3 & 2015 Feb 16 & WFC & 2700 & 6300 & 111 \\
\hline A746-1 & 2013 Jan 16 & SDSS & 3600 & 6200 & 110 \\
\hline A1240-1 & 2013 Dec 3 & SDSS & 2700 & 6850 & 120 \\
\hline A1240-2 & 2015 Feb 16 & SDSS & 2700 & 6820 & 164 \\
\hline A1612-1 & 2015 Feb 16 & SDSS & 1200 & 6750 & 186 \\
\hline A2034-1 & 2013 Jul 14 & SDSS & 2700 & 6700 & 158 \\
\hline A2443-1 & 2014 Jun 22 & SDSS & 2400 & 6400 & 153 \\
\hline A2443-2 & 2014 Jun 23 & SDSS & 2400 & 6400 & 163 \\
\hline A3365-1 & 2013 Jan 16 & WFC & 2700 & 6200 & 68 \\
\hline A3365-2 & 2013 Jan 16 & WFC & 2400 & 6200 & 66 \\
\hline A3365-3 & 2013 Dec 3 & WFC & 2700 & 6300 & 63 \\
\hline A3365-4 & 2015 Feb 16 & $\mathrm{SC}$ & 2700 & 6200 & 160 \\
\hline A3411-1 & 2013 Dec 3 & WFC & 2700 & 6650 & 132 \\
\hline A3411-2 & 2013 Dec 3 & WFC & 2700 & 6650 & 127 \\
\hline A3411-3 & 2013 Dec 4 & WFC & 2700 & 6650 & 128 \\
\hline A3411-4 & 2013 Dec 4 & WFC & 2700 & 6650 & 131 \\
\hline A3411-5 & 2015 Dec 13 & $\mathrm{SC}$ & 3600 & 6650 & 142 \\
\hline CIZAJ2242-1 & 2013 Jul 14 & WFC & 2700 & 6700 & 148 \\
\hline CIZAJ2242-2 & 2013 Jul 14 & WFC & 2700 & 6700 & 126 \\
\hline CIZAJ2242-3 & 2013 Sep 5 & $\mathrm{SC}$ & 2700 & 7000 & 90 \\
\hline CIZAJ2242-4 & 2013 Sep 5 & $\mathrm{SC}$ & 2700 & 7000 & 106 \\
\hline MACSJ1752-1 & 2013 Jul 14 & SDSS & 2700 & 6700 & 155 \\
\hline MACSJ1752-2 & 2013 Jul 14 & SDSS & 2700 & 6700 & 119 \\
\hline MACSJ1752-3 & 2013 Sep 5 & SDSS & 3600 & 7000 & 114 \\
\hline MACSJ1752-4 & 2013 Sep 5 & SDSS & 2700 & 7000 & 118 \\
\hline PLCKG287-1 & 2015 Feb 16 & $\mathrm{SC}$ & 3900 & 7950 & 207 \\
\hline PLCKG287-2 & 2015 Feb 16 & $\mathrm{SC}$ & 2700 & 7950 & 185 \\
\hline PLCKG287-3 & 2015 Feb 16 & SC & 2700 & 7950 & 193 \\
\hline PSZ1G108-1 & 2014 Jun 22 & DSS & 1800 & 7400 & 198 \\
\hline PSZ1G108-2 & 2014 Jun 23 & DSS & 1800 & 7650 & 168 \\
\hline RXCJ1053-1 & 2013 Jan 16 & SDSS & 2803 & 6200 & 113 \\
\hline RXCJ1053-2 & 2013 Dec 3 & SDSS & 2700 & 6200 & 84 \\
\hline RXCJ1053-3 & 2013 Dec 4 & SDSS & 2430 & 6200 & 98 \\
\hline RXCJ1314-1 & 2015 Feb 16 & SC & 2520 & 7120 & 196 \\
\hline RXCJ1314-2 & 2015 Feb 16 & SC & 2520 & 7120 & 207 \\
\hline ZwCl0008-1 & 2013 Jan 16 & WFC & 2063 & 6200 & 81 \\
\hline ZwCl0008-2 & 2013 Jul 14 & WFC & 2700 & 6700 & 81 \\
\hline ZwCl0008-3 & 2013 Sep 5 & WFC & 2700 & 7000 & 75 \\
\hline ZwCl0008-4 & 2013 Sep 5 & WFC & 3600 & 7000 & 73 \\
\hline ZwCl1447-1 & 2014 Jun 22 & SDSS & 1520 & 7850 & 149 \\
\hline ZwCl1447-2 & 2014 Jun 23 & SDSS & 1053 & 7850 & 138 \\
\hline ZwCl1856-1 & 2014 Jun 22 & DSS & 1800 & 7400 & 150 \\
\hline ZwCl1856-2 & 2014 Jun 23 & DSS & 1800 & 7400 & 101 \\
\hline $\mathrm{ZwCl} 2341-1$ & 2013 Jul 14 & SDSS & 2700 & 6700 & 130 \\
\hline $\mathrm{ZwCl} 2341-2$ & 2013 Jul 14 & SDSS & 2700 & 6700 & 131 \\
\hline ZwCl2341-3 & 2013 Sep 5 & SDSS & 2700 & 7000 & 148 \\
\hline
\end{tabular}

Note. Target imaging codes: WFC = Issac Newton Telescope Wide Field Camera presented in van Weeren et al. (2011c); SDSS = Sloan Digital Sky sSurvey (e.g., Alam et al. 2015); DSS = Palomar Observatory Digitized Sky Survey (Djorgovski et al. 1992); SC $=$ Subaru/SuprimeCam imaging (see Section 3.2).

We combine all known redshifts (from NED, the literature, and our DEIMOS survey) in the cluster fields and check for duplicates using the Topcat (Taylor 2005) software using the sky function with a $1^{\prime \prime}$ tolerance. We also checked for self-consistency between our survey and the literature by computing the median difference of galaxies with multiple redshift estimates for each cluster. Such offsets between the inferred line-of-sight velocity differences are due to, e.g., 
Table 5

Breakdown of Spectroscopy from Our DEIMOS Survey and the Literature

\begin{tabular}{|c|c|c|c|c|}
\hline Cluster & $\begin{array}{c}\text { DEIMOS } \\
\text { Galaxies }\end{array}$ & $\begin{array}{c}\text { Unique } \\
\text { Literature }\end{array}$ & $\begin{array}{c}\text { Cluster } \\
\text { members }\end{array}$ & References \\
\hline 1RXSJ0603 & 311 & 0 & 242 & ... \\
\hline A115 & 237 & 76 & 198 & $\begin{array}{c}\text { B83, Z90, } \\
\text { B07, } \\
\text { 2MASS, } \\
\text { SDSS }\end{array}$ \\
\hline A521 & 0 & 193 & 126 & M00, F03 \\
\hline A523 & 246 & 61 & 149 & G16 \\
\hline A746 & 94 & 6 & 66 & $\begin{array}{c}\text { 2MASS, } \\
\text { SDSS }\end{array}$ \\
\hline A781 & 0 & 875 & 435 & G05, SDSS \\
\hline A1240 & 188 & 151 & 146 & $\begin{array}{c}\text { B09, } \\
\text { 2MASS, } \\
\text { SDSS }\end{array}$ \\
\hline A1300 & 0 & 270 & 227 & P97, Z12 \\
\hline A1612 & 80 & 39 & 73 & SDSS \\
\hline A2034 & 125 & 129 & 139 & SDSS, O14 \\
\hline A2061 & 0 & 404 & 157 & SDSS \\
\hline A2163 & 0 & 407 & 382 & M08 \\
\hline A2255 & 0 & 406 & 270 & SDSS \\
\hline A2345 & 0 & 103 & 101 & B10 \\
\hline A2443 & 247 & 17 & 156 & SDSS \\
\hline A2744 & 0 & 695 & 380 & C87, B06, 011 \\
\hline A 3365 & 246 & 33 & 150 & $\mathrm{~K} 98,6 \mathrm{dF}$ \\
\hline A3411 & 316 & 0 & 242 & vW17 \\
\hline CIZAJ2242 & 257 & 0 & 217 & D15 \\
\hline MACSJ1149 & 0 & 591 & 258 & SDSS, E14 \\
\hline MACSJ1752 & 397 & 0 & 176 & $\cdots$ \\
\hline PLCKG287 & 337 & 317 & 305 & $\mathrm{a}$ \\
\hline PSZ1G108 & 60 & 0 & 40 & $\ldots$ \\
\hline RXCJ1053 & 224 & 144 & 119 & SDSS \\
\hline RXCJ1314 & 277 & 18 & 156 & V02 \\
\hline ZwCl0008 & 203 & 0 & 116 & G17 \\
\hline ZwCl1447 & 200 & 0 & 116 & $\cdots$ \\
\hline ZwCl1856 & 69 & 0 & 47 & $\cdots$ \\
\hline ZwCl2341 & 317 & 62 & 224 & SDSS, B13 \\
\hline
\end{tabular}

Note.

a 317 unique galaxy redshifts were obtained from VLT VIMOS Obs ID. 094. A-0529, PI M. Nonino. We have included them in our redshift analysis, but we will not publish them in the Appendix. Column 1: cluster names. Column 2: number of spectroscopically confirmed galaxies (including foreground and background in our DEIMOS survey). Column 3: unique literature galaxies within $5 \mathrm{Mpc}$ of the cluster center and $10,000 \mathrm{~km} \mathrm{~s}^{-1}$ of the cluster redshift. Column 4: number of cluster members in redshift analysis. Column 5: reference codes: B83 = Beers et al. (1983), Z90 = Zabludoff et al. (1990), B07 = Barrena et al. (2007), 2MASS = Skrutskie et al. (2006), SDSS = Alam et al. (2015), M00 = Maurogordato et al. (2000), F03 = Ferrari et al. (2003), G16 = Girardi et al. (2016), G05 = Geller et al. (2005), B09 = Barrena et al. (2009), P97 = Pierre et al. (1997), Z12 = Ziparo et al. (2012), O14 = Owers et al. (2014), M08 = Maurogordato et al. (2008), B10 $=$ Boschin et al. (2010), C87 = Couch \& Sharples (1987), B06 $=$ Boschin et al. (2006), O11 = Owers et al. (2011), K98 = Katgert et al. (1998), $6 \mathrm{dF}=$ Jones et al. (2005), $\mathrm{vW} 17=$ van Weeren et al. (2017), D15 = Dawson et al. (2015), E14 = Ebeling et al. (2014), V02 = Valtchanov et al. (2002), G17 = Golovich et al. (2017), B13 = Boschin et al. (2013).

differing wavelength calibration. We shifted literature offsets to our derived redshift estimates based on this analysis, but we note that none of the offsets were within the $1 \sigma$ estimate for the cluster redshift for any cluster, so our results are not dependent on these corrections.

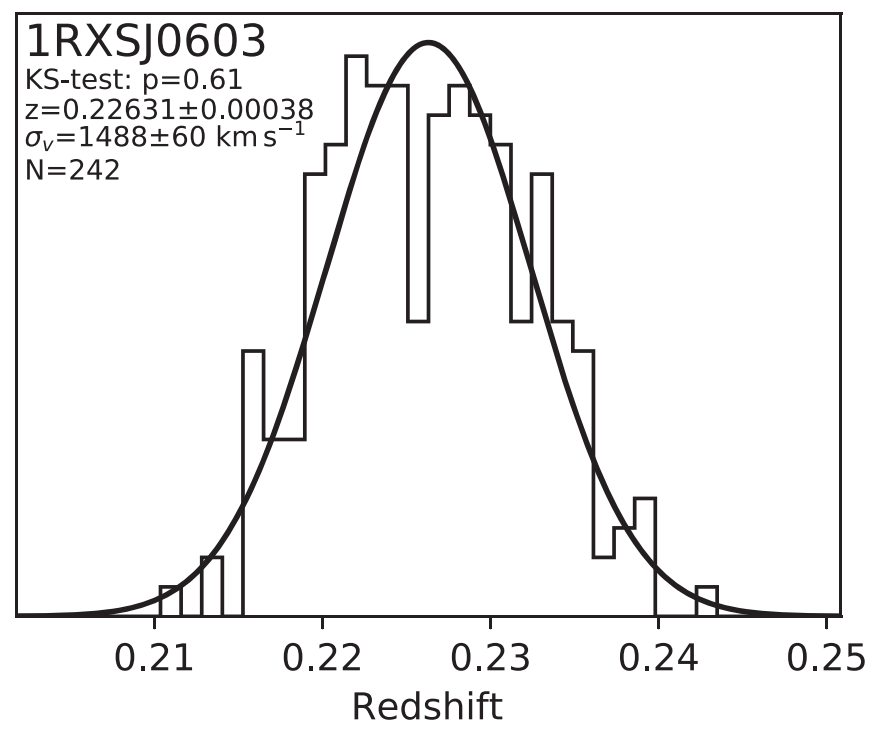

Figure 4. Redshift distribution for 1 RXSJ0603 based on our DEIMOS spectroscopic survey. Galaxies are selected with a shrinking 3D aperture until a stable set of galaxies within $R_{200}$ and $\pm 3 \sigma_{v}$ is achieved. The global redshift analysis using the biweight statistic and bias-corrected $68 \%$ confidence limits are presented in the panel. The p-value for a KS test for Gaussianity is presented as well. The panel width is $12,000 \mathrm{~km} \mathrm{~s}^{-1}$ centered on the cluster redshift. Bins are $300 \mathrm{~km} \mathrm{~s}^{-1}$ at the cluster redshift. The complete figure set (29 images) is available in the online journal.

(The complete figure set (29 images) is available.)

These combined catalogs of unique spectroscopically confirmed objects are studied in Section 4. In Table 5, a breakdown of the numbers of spectroscopic redshifts from the literature review and DEIMOS survey are reported.

\section{Redshift Analysis}

In this section we describe the process of selecting spectroscopic cluster members from our combined redshift catalogs (see Tables 4 and 5).

\subsection{Spectroscopic Catalog Generation}

We cut each spectroscopic catalog to only include objects within $R_{200}$ in projected space and to within $\bar{v} \pm 3 \sigma_{v}$, where $\bar{v}$ is the average line-of-sight velocity and $\sigma_{v}$ is the cluster velocity dispersion. This is accomplished with an iterative process starting with $5 \mathrm{Mpc}$ and $10,000 \mathrm{~km} \mathrm{~s}^{-1}$ and shrinking the radius and velocity window until an equilibrium catalog is achieved. The radius is determined in each step by translating the velocity dispersion into a mass using the Evrard et al. (2008) scaling relations followed by estimating $R_{200}$ based on the mass.

This reduces the chance of inclusion of galaxies that are uninvolved in the merger. An instructive example is A2061, where A2067 is $\sim 2.7 \mathrm{Mpc}\left(30^{\prime}\right)$ to the northeast and at a similar redshift, but uninvolved in the merger. The iterative shrinking aperture was able to eliminate galaxies from A2067 from the redshift catalog despite being at a similar redshift because it is outside of $R_{200}$. A second example is A523 $(z \sim 0.1)$, which has two background groups at $z \sim 0.14$ within $R_{200}$ in projection (Girardi et al. 2016). 


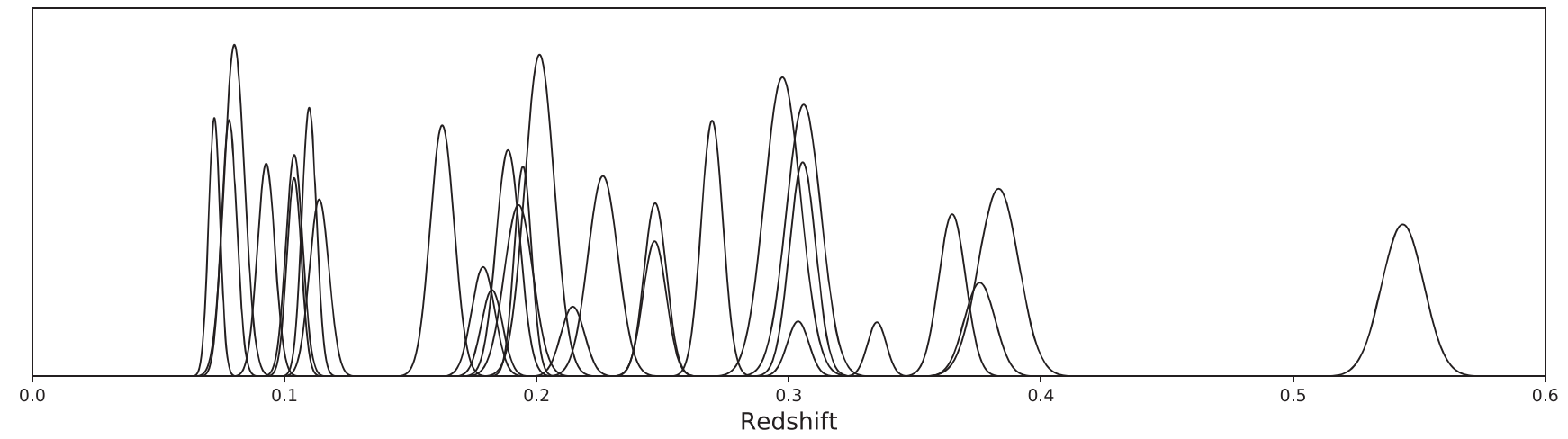

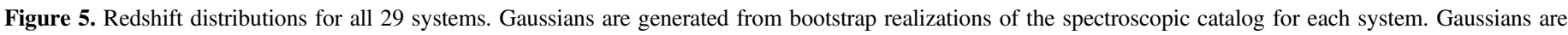
scaled such that their areas are proportional to the number of spectroscopic galaxies for each system.

After this process, 5413 spectroscopic galaxies remain across the 29 systems. The breakdown for each cluster is presented in Table 5.

\subsection{One-dimensional Redshift Analysis}

We display the one-dimensional redshift distribution for 1RXSJ0603 in Figure 4. An analogous figure for the remaining 28 systems is available in the online version as a figure set. The corresponding normalized Gaussian distribution is overlaid with the cluster redshift and velocity dispersion given by the biweight and bias-corrected 68\% confidence intervals. We implement the biweight statistic based on 10,000 bootstrap samples of the member galaxies and calculate the biascorrected $68 \%$ confidence limits for the redshift and velocity dispersion from the bootstrap sample. This method is more robust to outliers than the dispersion of the Gaussians generated by our statistical model (Beers et al. 1990).

We test the goodness of fit of the corresponding Gaussian distribution using a Kolmogorov-Smirnov (KS) test. The results of this analysis are displayed in Figure 4 and in the online figure set for the other systems. We generally find good agreement between the spectroscopic data and single Gaussian distributions, which implies that the merging subclusters have line-of-sight velocity differences that are small compared to the velocity dispersion. The lowest p-value for the KS test is 0.007 for A781, which is known to be composed of several subclusters with large velocity differences (Geller et al. 2005). In Figure 5, the 29 resulting Gaussians are presented to demonstrate the sample distribution of spectroscopic cluster members. The area of a given Gaussian is proportional to the population of galaxies in the respective cluster catalogs.

We also fit increasing numbers of Gaussians to the onedimensional redshift distributions of each cluster utilizing an expectation-maximization Gaussian mixture model method from the Sci-Kit Learn python module. We varied the number of Gaussians from one to seven for each cluster. A single Gaussian model was strongly preferred for 27 of the 29 clusters according to the Bayesian Information Criterion. For A3365, the one Gaussian model was only slightly favored over a two Gaussian model, and for A781, a two-halo model was strongly preferred.

In a second paper (Golovich et al. 2018), we studied the three-dimensional distribution of galaxies and determine substructure and merger scenarios using a panchromatic data set.

\section{Discussion}

\subsection{Analysis of the Spectroscopic Survey}

In Section 3.3.1 we discuss our methods of selecting targets for our spectroscopic survey. Because our photometric survey was ongoing during this process, we utilized the best available photometry for spectroscopic targeting (see Table 4). Here, we analyze the success of the various targeting methods. Broadly, two distinct methods for selecting potential targets were implemented. For 21 of 54 slit-masks, potential targets were identified via a photometric redshift selection based on SDSS photometric redshifts. For the remaining 33 slit-masks, a red sequence selection was implemented; however, the quality of the imaging (seeing and depth) varied substantially depending on the source. In Table 4, the spectra are broken down by individual slit-mask, targeting method, imaging used for targeting, and redshift.

The biggest indication of the effect of the target imaging quality on the spectroscopic survey is with the fraction of targeted objects that yielded a secure redshift of a cluster member, which was our primary goal. In Table 6 , the $\sim 7000$ targeted objects are broken down by the type of object detected. Across the survey, $77 \%$ of all targeted objects yielded a secure redshift estimate. Of these, $49 \%$ were cluster galaxies. The largest sources of contaminants were background galaxies $(26 \%)$ and stars $(18 \%)$.

Background galaxies were detected at higher frequency with photometric redshift targeting. Detection of these objects also decreased with the redshift of the cluster. A substantial fraction of stars were detected in a few fields that had either sub-par imaging for target selection, or had low galactic latitude. The effect of imaging quality with regards to stellar contamination is evident in the five A3411 slit-masks. The first four were observed using INT/WFC targeting, and the fraction of stars increased for successive slit-masks as the best member candidates were depleted by earlier masks. For the fifth slitmask, Subaru/SuprimeCam was utilized for targeting, and the fraction of stars decreased substantially. The trade-off was a larger fraction of background galaxies, which is explained by the increased depth of the imaging and also by the fact that the four previous slit-masks had identified many of the brightest cluster members. One benefit of the background galaxy redshift determination is for developing training sets for weak-lensing source selection. Furthermore, gravitational lensing is complicated by massive structures along the line of sight, and overdensities of background galaxies may help reveal these types of massive background structures; however, the 
Table 6

Breakdown of Detected Objects for the DEIMOS Spectroscopic Survey

\begin{tabular}{|c|c|c|c|c|c|c|}
\hline Slit-mask & $\%$ Secure & $\%$ Stars & $\%$ Cluster & $\%$ Foreground & $\%$ Background & \# Serendips \\
\hline 1RXSJ0603-1 & 88 & 14 & 59 & 3 & 11 & 14 \\
\hline 1RXSJ0603-2 & 86 & 13 & 59 & 1 & 13 & 7 \\
\hline 1RXSJ0603-3 & 88 & 11 & 64 & 2 & 10 & 15 \\
\hline 1RXSJ0603-4 & 87 & 22 & 51 & 5 & 10 & 11 \\
\hline A115-1 & 78 & 7 & 48 & 9 & 15 & 2 \\
\hline A115-2 & 80 & 4 & 42 & 6 & 30 & 3 \\
\hline A523-1 & 82 & 2 & 49 & 3 & 27 & 10 \\
\hline A523-2 & 80 & 1 & 37 & 3 & 38 & 6 \\
\hline A523-3 & 83 & 5 & 34 & 1 & 43 & 5 \\
\hline A746-1 & 87 & 2 & 60 & 5 & 22 & 2 \\
\hline A1240-1 & 72 & 2 & 41 & 3 & 25 & 5 \\
\hline A1240-2 & 68 & 4 & 27 & 4 & 33 & 3 \\
\hline A1612-1 & 50 & 6 & 30 & 3 & 10 & 5 \\
\hline A2034-1 & 80 & 2 & 39 & 3 & 37 & 3 \\
\hline A2443-1 & 83 & 1 & 58 & 1 & 24 & 5 \\
\hline A2443-2 & 79 & 3 & 39 & 5 & 31 & 7 \\
\hline A3365-1 & 91 & 9 & 49 & 0 & 31 & 2 \\
\hline A3365-2 & 87 & 12 & 39 & 0 & 35 & 4 \\
\hline A $3365-3$ & 79 & 6 & 33 & 0 & 40 & 2 \\
\hline A3365-4 & 61 & 3 & 14 & 0 & 45 & 1 \\
\hline A3411-1 & 77 & 25 & 46 & 0 & 5 & 1 \\
\hline A3411-2 & 81 & 43 & 25 & 1 & 12 & 4 \\
\hline A3411-3 & 93 & 28 & 43 & 0 & 11 & 2 \\
\hline A3411-4 & 90 & 58 & 20 & 0 & 12 & 2 \\
\hline A3411-5 & 76 & 12 & 37 & 0 & 27 & 2 \\
\hline CIZAJ2242-1 & 74 & 18 & 49 & 2 & 4 & 12 \\
\hline CIZAJ2242-2 & 77 & 24 & 48 & 2 & 2 & 25 \\
\hline CIZAJ2242-3 & 79 & 36 & 29 & 7 & 8 & 23 \\
\hline CIZAJ2242-4 & 86 & 25 & 50 & 3 & 7 & 22 \\
\hline MACSJ1752-1 & 81 & 8 & 47 & 14 & 15 & 3 \\
\hline MACSJ1752-2 & 84 & 8 & 49 & 14 & 13 & 4 \\
\hline MACSJ1752-3 & 83 & 9 & 32 & 13 & 29 & 9 \\
\hline MACSJ1752-4 & 84 & 8 & 41 & 17 & 18 & 2 \\
\hline PLCKG287-1 & 69 & 0 & 47 & 23 & 7 & 1 \\
\hline PLCKG287-2 & 71 & 3 & 45 & 12 & 10 & 2 \\
\hline PLCKG287-3 & 41 & 3 & 18 & 12 & 8 & 0 \\
\hline PSZ1G108-1 & 75 & 53 & 14 & 6 & 2 & 2 \\
\hline PSZ1G108-2 & 85 & 73 & 8 & 2 & 2 & 2 \\
\hline RXCJ1053-1 & 72 & 0 & 17 & 2 & 53 & 2 \\
\hline RXCJ1053-2 & 89 & 7 & 38 & 0 & 44 & 0 \\
\hline RXCJ1053-3 & 77 & 1 & 27 & 1 & 46 & 1 \\
\hline RXCJ1314-1 & 79 & 4 & 45 & 6 & 24 & 3 \\
\hline RXCJ1314-2 & 64 & 0 & 27 & 4 & 32 & 3 \\
\hline ZwCl0008-1 & 74 & 6 & 53 & 0 & 14 & 5 \\
\hline ZwCl0008-2 & 79 & 23 & 38 & 0 & 16 & 10 \\
\hline ZwCl0008-3 & 77 & 23 & 32 & 0 & 23 & 14 \\
\hline ZwCl0008-4 & 89 & 27 & 19 & 4 & 37 & 17 \\
\hline ZwCl1447-1 & 77 & 1 & 49 & 13 & 14 & 1 \\
\hline ZwCl1447-2 & 65 & 2 & 30 & 16 & 16 & 3 \\
\hline ZwCl1856-1 & 83 & 54 & 22 & 5 & 4 & 1 \\
\hline ZwCl1856-2 & 85 & 64 & 15 & 5 & 1 & 3 \\
\hline ZwCl2341-1 & 71 & 0 & 43 & 4 & 23 & 7 \\
\hline $\mathrm{ZwCl2341-2}$ & 77 & 2 & 46 & 6 & 23 & 4 \\
\hline $\mathrm{ZwCl} 2341-3$ & 82 & 2 & 44 & 5 & 30 & 1 \\
\hline \multicolumn{7}{|l|}{ Targeting Method } \\
\hline Photometric Redshifts & 76 & 4 & 41 & 7 & 25 & 72 \\
\hline Color-Magnitude & 77 & 21 & 35 & 4 & 16 & 233 \\
\hline \multicolumn{7}{|l|}{ Imaging } \\
\hline WFC & 83 & 20 & 43 & 1 & 18 & 168 \\
\hline SDSS & 76 & 4 & 41 & 7 & 25 & 72 \\
\hline $\mathrm{SC}$ & 67 & 7 & 35 & 8 & 19 & 57 \\
\hline DSS & 81 & 60 & 14 & 5 & 2 & 8 \\
\hline
\end{tabular}


Table 6

(Continued)

\begin{tabular}{lcccccc}
\hline \hline Slit-mask & \% Secure & \% Stars & \% Cluster & \% Foreground & \% Background & \# Serendips \\
\hline Cluster Redshifts & & & & & & \\
\hline$z<0.1$ & 76 & 5 & 28 & 3 & 43 \\
$0.1<z<0.2$ & 78 & 15 & 39 & 4 & 20 & 12 \\
$0.2<z<0.3$ & 79 & 6 & 37 & 12 & 22 & 10 \\
$z>0.3$ & 74 & 14 & 38 & 5 & 33 & 20 \\
\hline Totals & 77 & 22 & 305 & \\
\hline
\end{tabular}

Note. The percentages of stars, cluster members, and foreground and background objects may not add to the total percentage of secure objects due to rounding. Column 1: cluster and slit-mask number. Column 2: percentage of secure redshifts among targeted objects. Column 3-6: percentage of stars, cluster member galaxies, foreground galaxies, and background galaxies, respectively. Column 7: number of serendipitous detections.

spectroscopic survey was not designed to detect such systems, so any such detection is serendipitous. Foreground galaxies accounted for only $6 \%$ of secure redshifts, and these were predominantly detected in higher-redshift cluster fields. Finally, 305 objects were detected serendipitously; i.e., a single slit had one or more traces in addition to the targeted object. These were predominantly detected in low-galactic -latitude fields and were composed of stars, although, $\sim 50$ cluster galaxies were detected in this manner across the survey.

\subsection{Cluster Redshift Histograms}

The presence of radio relics in merging galaxy clusters constitutes a strong prior for ongoing merging activity. Given this, these 29 merging clusters are expected to be composed of 2 or more subclusters. However, 28 of the 29 systems are well fit by a single Gaussian $(p>0.05)$.

There are two potential explanations for this, which are not mutually exclusive: 1) radio relics indicate a merger occurring within the plane of the sky (transverse to the line of sight), and/ or 2) radio relics indicate a merger observed near-apocenter. Based on the redshift results alone, both scenarios are plausible. First, most of our relics were detected in shallow surveys, and the surface brightness is higher when the line of sight intersects a large fraction of the emission in three dimensions (Skillman et al. 2013). Furthermore, detected radio relics have been shown to be highly polarized (e.g Govoni \& Feretti 2004; Ferrari et al. 2008), which correlates with a transverse viewing angle of the merger (Ensslin et al. 1998). Second, radio relics occur for only a small fraction of the full merger phase, and it takes time for the radio relic to develop (see Figure 5 of Skillman et al. 2013). This may explain why the Bullet Cluster's bow shock is not coincident with a bright radio relic. Meanwhile, El Gordo contains radio relics, and it was shown to be returning from apocenter ( $\mathrm{Ng}$ et al. 2015). Thus, it is likely a combination of the two scenarios that explain the unimodal redshift distributions in 28 of 29 systems in the sample. Recent magnetohydrodynamical simulations suggest that explanation (1) is more likely for radio-relic systems (Vazza et al. 2012; Wittor et al. 2017)

The one outlier, A781, is known to be composed of multiple clusters at various redshifts (Geller et al. 2005). The system is composed of two clusters in projection at $z \sim 0.3$ and $z \sim 0.42$. Here, we studied the $z \sim 0.3$ system, which is further split into two redshift peaks (see the online figure analogous to Figure 4. The radio relic is associated with the slightly higher redshift peak on the western side of the cluster. The lower-redshift peak is associated with an infalling subcluster, which is yet to merge,

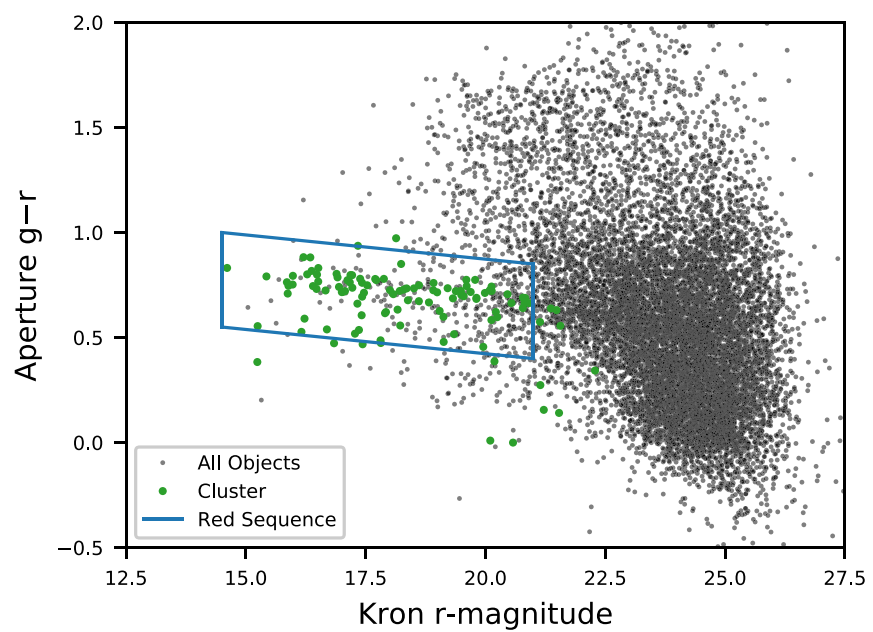

Figure 6. Color-magnitude diagram for the photometric catalog of RXCJ1053 with overlaid spectroscopic matches. The red sequence selection box is shown in blue.

based on the undisturbed X-ray surface brightness distribution (see Figure 1 of Sehgal et al. 2008, where this subcluster is referred to as the Middle subcluster).

\subsection{Potential Uses for These Data}

The photometric data presented in this paper are sufficiently deep for detailed, wide-field, weak gravitational lensing analyses of each cluster (see Bartelmann \& Schneider 2001; Hoekstra 2013, for a review). This will allow for the mapping of the total mass distribution of each system, as well as allow for mass estimation of each subcluster in a manner unbiased by the dynamical interaction of the merger (e.g., Jee et al. 2015, 2016). Both galaxy velocity dispersion based mass estimates assuming the system is virialized (Takizawa et al. 2010) and X-ray temperature or luminosity scaling relation based mass estimates assuming the cluster is in hydrostatic equilibrium (Zhang et al. 2010) overestimate the mass in merging systems. Takizawa et al. (2010), however, showed that mergers along the line of sight are more strongly affected by this, and most strongly near core passage. Radio-relic systems are typically observed $\sim 1$ Gyr after pericentric passage ( $\mathrm{Ng}$ et al. 2015; Golovich et al. 2016, 2017; van Weeren et al. 2017).

Because we took images with two photometric filters that straddle the $4000 \AA$ break, colors may be assigned to objects, allowing color-magnitude selection. This allows for selection 
Table 7

DEIMOS Spectroscopic Survey Catalog

\begin{tabular}{|c|c|c|c|c|c|c|c|c|}
\hline ID & R.A. & Decl. & $g$ & $r$ & $i$ & $z$ & $\sigma_{z}$ & Spectral Features \\
\hline 1 & 90.84466369 & 42.27306837 & 20.28 & 18.81 & 17.78 & 0.220011 & $3.81 \mathrm{E}-05$ & $\mathrm{Hb}$ ab, Mg I (b), [Fe I], Na I (D), Ha ab \\
\hline 1 & 90.81274054 & 42.25876563 & 23.58 & 22.16 & 21.12 & 0.508420 & $3.06 \mathrm{E}-05$ & $\operatorname{Mg} \mathrm{I}(\mathrm{b}),[\mathrm{Fe} \mathrm{I}] \mathrm{m} \mathrm{Na} \mathrm{I}(\mathrm{D}), \mathrm{Ha}$ \\
\hline 1 & 90.90432650 & 42.12064156 & 21.90 & 20.36 & 19.32 & 0.224067 & $3.92 \mathrm{E}-05$ & $G$ band, $\mathrm{Hb}$ ab, $\mathrm{Mg} \mathrm{I}(\mathrm{b}),[\mathrm{Fe} \mathrm{I}]$ \\
\hline 1 & 90.84777475 & 42.17517749 & 21.20 & 19.71 & 18.71 & 0.225441 & 3.92E-05 & $G$ band, $\mathrm{Mg} \mathrm{I}$ (b), [Fe I], Na I (D) \\
\hline 1 & 90.80537365 & 42.16394040 & 22.49 & 21.05 & 20.08 & 0.227767 & 3.99E-05 & $\mathrm{Hb} a b, \mathrm{Mg}$ I (b), Na I (D), Ha ab \\
\hline
\end{tabular}

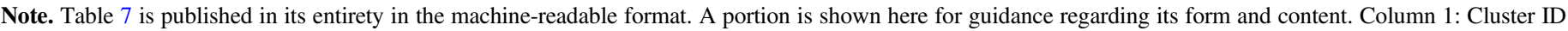

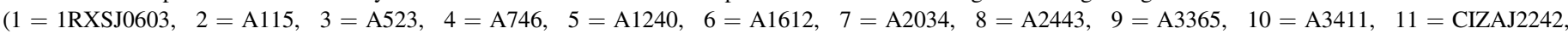

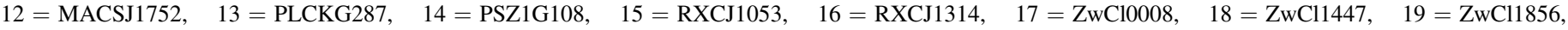

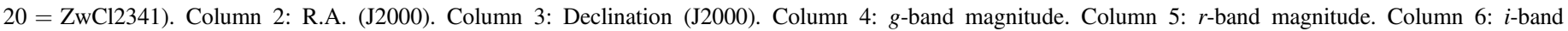
magnitude. Column 7: redshift; Column 8: redshift uncertainty. Column 9: spectral features identified in 1D spectrum.

(This table is available in its entirety in machine-readable form.)

of background galaxies for weak lensing as well as cluster members based on the red sequence technique (see Figure 6).

The spectroscopic data contain the added information of line-of-sight motion, which allows for a pure catalog of cluster members. From this catalog, dynamical modeling of the mergers may be achieved. Merging clusters are efficient astrophysical laboratories for studying several phenomena including particle acceleration, cool-core disruption, and potential self-interacting DM signals. Many of these are timedependent and velocity-dependent, which requires accurate dynamical models. Furthermore, these dynamical models are invaluable for simulators in the form of constrained initial conditions. Finally, the spectral quality from DEIMOS allows for analyses of merging-induced star formation, galaxy evolution, and AGN activity (see, e.g., Sobral et al. 2015).

\subsection{Summary}

In this paper, we presented our observational strategy, reduction, and analysis of $\sim 20 \mathrm{hr}$ of Subaru/SuprimeCam imaging of 29 merging galaxy clusters alongside our spectroscopic follow-up of 7000 objects (54 slit-masks) with Keck/ DEIMOS. We presented $\sim 4340$ new high-quality galaxy redshifts and spectral features from our spectroscopic survey matched to the photometry from our Subaru/SuprimeCam survey in Table 7. These data are combined with literature spectroscopy and SuprimeCam imaging, which resulted in 5297 cluster members in total across the 29 systems. A onedimensional redshift analysis showed that 28 of 29 of the systems are well fit by a single Gaussian distribution. This suggests the ongoing mergers are occurring either within the plane of the sky or are observed near-apocenter (or a combination of the two factors). We analyzed the effect of different imaging sources and selection methods for targeting slits in our spectroscopic survey, and we discussed possible uses for this large data set of photometric and spectroscopic observations of galaxies within merging galaxy clusters.

We would like to thank the broader membership of the Merging Cluster Collaboration for their continual development of the science motivating this work, for useful conversations, and for diligent proofreading, editing, and feedback. This material is based upon work supported by the National Science Foundation under grant No. (1518246). This material is based in part upon work supported by STSci grant HST-GO-13343.001-A. Part of this was work performed under the auspices of the U.S. DOE by LLNL under Contract DE-AC52-07NA27344. Some of the data presented herein were obtained at the W.M. Keck Observatory, which is operated as a scientific partnership among the California Institute of Technology, the University of California and the National Aeronautics and Space Administration. The Observatory was made possible by the generous financial support of the W.M. Keck Foundation. This work is based in part on data collected at Subaru Telescope, which is operated by the National Astronomical Observatory of Japan. Funding for the Sloan Digital Sky Survey IV has been provided by the Alfred P. Sloan Foundation, the U.S. Department of Energy Office of Science, and the Participating Institutions. SDSS acknowledges support and resources from the Center for High-Performance Computing at the University of Utah. The SDSS website is www.sdss.org. The Digitized Sky Surveys were produced at the Space Telescope Science Institute under U.S. Government grant NAG W-2166. Funding for the DEEP2/DEIMOS pipelines has been provided by NSF grant AST-0071048. The DEIMOS spectrograph was funded by grants from CARA (Keck Observatory) and UCO/Lick Observatory, a NSF Facilities and Infrastructure grant (ARI92-14621), the Center for Particle Astrophysics, and by gifts from Sun Microsystems and the Quantum Corporation. This research has made use of the NASA/ IPAC Extragalactic Database (NED), which is operated by the Jet Propulsion Laboratory, California Institute of Technology, under contract with the National Aeronautics and Space Administration. This research has made use of NASA's Astrophysics Data System. This work i based in part on data collected at Subaru Telescope and obtained from the SMOKA, which is operated by the Astronomy Data Center, National Astronomical Observatory of Japan. M.J.J. acknowledges support for the current research from the National Research Foundation of Korea under the programs 2017R1A2B2004644 and 2017R1A4A1015178.

Facility: Keck (DEIMOS) INT (WFC) Subaru (SuprimeCam) VLT (VIMOS).

\section{Appendix Spectroscopic Catalog}

Table 7 contains the R.A. and Decl. coordinates, redshifts, Subaru/SuprimeCam magnitudes, and spectral features for 4431 galaxies identified by our DEIMOS spectroscopic survey (see Section 3.3). Each spectroscopically confirmed object was matched with the Subaru/SuprimeCam catalog (see Section 3.2.2) using the Topcat software (Taylor 2005) with a $1^{\prime \prime}$ tolerance. Objects without photometric matches were discarded. Photometric objects were matched to their nearest spectroscopic match and were not allowed to match more than once. 


\section{ORCID iDs}

N. Golovich (1) https://orcid.org/0000-0003-2632-572X

W. A. Dawson (1) https://orcid.org/0000-0003-0248-6123

D. M. Wittman (1) https://orcid.org/0000-0002-0813-5888

B. Benson (1) https://orcid.org/0000-0002-6099-4989

R. J. van Weeren (1) https://orcid.org/0000-0002-0587-1660

F. Andrade-Santos (i) https://orcid.org/0000-0002-8144-9285

D. Sobral (1) https://orcid.org/0000-0001-8823-4845

F. de Gasperin (i) https://orcid.org/0000-0003-4439-2627

M. Bradač (i) https://orcid.org/0000-0001-5984-0395

A. Peter 1 h https://orcid.org/0000-0002-8040-6785

\section{References}

Adelman-McCarthy, J. K., Agüeros, M. A., Allam, S. S., et al. 2007, ApJS, 172,634

Alam, S., Albareti, F. D., Allende Prieto, C., et al. 2015, ApJS, 219, 12

Baba, H., Yasuda, N., Ichikawa, S.-I., et al. 2002, in ASP Conf. Ser. 281, Astronomical Data Analysis Software and Systems XI, ed. D. A. Bohlender, D. Durand, \& T. H. Handley (San Francisco, CA: ASP), 298

Baldwin, J. A., Phillips, M. M., \& Terlevich, R. 1981, PASP, 93, 5

Ballarati, B., Feretti, L., Ficarra, A., et al. 1981, A\&A, 100, 323

Barrena, R., Boschin, W., Girardi, M., \& Spolaor, M. 2007, A\&A, 469, 861

Barrena, R., Girardi, M., Boschin, W., \& Dasí, M. 2009, A\&A, 503, 357

Bartelmann, M., \& Schneider, P. 2001, PhR, 340, 291

Beers, T. C., Flynn, K., \& Gebhardt, K. 1990, AJ, 100, 32

Beers, T. C., Geller, M. J., \& Huchra, J. P. 1983, ApJ, 264, 356

Benítez, N. 2000, ApJ, 536, 571

Benson, B., Wittman, D. M., Golovich, N., et al. 2017, ApJ, 841, 7

Bertin, E. 2006, in ASP Conf. Ser. 351, Astronomical Data Analysis Software and Systems XV, ed. C. Gabriel (San Francisco, CA: ASP), 112

Bertin, E., \& Arnouts, S. 1996, A\&AS, 117, 393

Bertin, E., Mellier, Y., Radovich, M., et al. 2002, in ASP Conf. Ser.281, Astronomical Data Analysis Software and Systems XI, ed. D. A. Bohlender, D. Durand, \& T. H. Handley (San Francisco, CA: ASP), 228

Blandford, R., \& Eichler, D. 1987, PhR, 154, 1

Bolzonella, M., Miralles, J.-M., \& Pelló, R. 2000, A\&A, 363, 476

Bonafede, A., Brüggen, M., van Weeren, R., et al. 2012, MNRAS, 426, 40

Boschin, W., Barrena, R., \& Girardi, M. 2010, A\&A, 521, A78

Boschin, W., Girardi, M., \& Barrena, R. 2013, MNRAS, 434, 772

Boschin, W., Girardi, M., Spolaor, M., \& Barrena, R. 2006, A\&A, 449, 461

Brunetti, G., Giacintucci, S., Cassano, R., et al. 2008, Natur, 455, 944

Brunetti, G., \& Jones, T. W. 2014, IJMPD, 23, 1430007

Buton, C., Copin, Y., Aldering, G., et al. 2013, A\&A, 549, A8

Cavaliere, A., \& Fusco-Femiano, R. 1976, A\&A, 49, 137

Chung, S. M., Gonzalez, A. H., Clowe, D., et al. 2009, ApJ, 691, 963

Clowe, D., Bradac, M., Gonzalez, A., et al. 2006, ApJL, 648, L109

Coe, D., Umetsu, K., Zitrin, A., et al. 2012, ApJ, 757, 22

Condon, J. J., Cotton, W. D., Greisen, E. W., et al. 1998, AJ, 115, 1693

Couch, W. J., \& Sharples, R. M. 1987, MNRAS, 229, 423

Dawson, W. A. 2013, ApJ, 772, 131

Dawson, W. A., Jee, M. J., Stroe, A., et al. 2015, ApJ, 805, 143

Dawson, W. A., Wittman, D., Jee, M. J., et al. 2012, ApJL, 747, L42

de Gasperin, F., Intema, H. T., van Weeren, R. J., et al. 2015a, MNRAS, 453,3483

de Gasperin, F., Ogrean, G. A., van Weeren, R. J., et al. 2015b, MNRAS, 448, 2197

de Gasperin, F., van Weeren, R. J., Brüggen, M., et al. 2014, MNRAS, 444, 3130

Djorgovski, S., Lasker, B. M., Weir, W. N., et al. 1992, BAAS, 24, 750

Duffy, A. R., Schaye, J., Kay, S. T., \& Dalla Vecchia, C. 2008, MNRAS, 390, L64

Ebeling, H., Ma, C.-J., \& Barrett, E. 2014, ApJS, 211, 21

Ensslin, T. A., Biermann, P. L., Klein, U., \& Kohle, S. 1998, A\&A, 332, 395

Evrard, A. E., Bialek, J., Busha, M., et al. 2008, ApJ, 672, 122

Faber, S. M., Phillips, A. C., Kibrick, R. I., et al. 2003, Proc. SPIE, 4841, 1657 Feretti, L., Giovannini, G., Govoni, F., \& Murgia, M. 2012, A\&ARv, 20, 54

Ferrari, C., Govoni, F., Schindler, S., Bykov, A. M., \& Rephaeli, Y. 2008, SSRv, 134, 93

Ferrari, C., Maurogordato, S., Cappi, A., \& Benoist, C. 2003, A\&A, 399, 813 Geller, M. J., Dell'Antonio, I. P., Kurtz, M. J., et al. 2005, ApJL, 635, L125
Girardi, M., Boschin, W., Gastaldello, F., et al. 2016, MNRAS, 456, 2829 Golovich, N., Dawson, W. A., Wittman, D., et al. 2016, ApJ, 831, 110

Golovich, N., Dawson, W. A., Wittman, D. M., et al. 2018, ApJ, submitted (arXiv:1806.10619)

Golovich, N., van Weeren, R. J., Dawson, W. A., Jee, M. J., \& Wittman, D. 2017, ApJ, 838, 110

Govoni, F., \& Feretti, L. 2004, IJMPD, 13, 1549

Hoekstra, H. 2013, arXiv:1312.5981

Jee, M. J., Dawson, W. A., Stroe, A., et al. 2016, ApJ, 817, 179

Jee, M. J., Stroe, A., Dawson, W., et al. 2015, ApJ, 802, 46

Jester, S., Schneider, D. P., Richards, G. T., et al. 2005, AJ, 130, 873

Jones, D. H., Saunders, W., Read, M., \& Colless, M. 2005, PASA, 22, 277

Kahlhoefer, F., Schmidt-Hoberg, K., Frandsen, M. T., \& Sarkar, S. 2014, MNRAS, 437, 2865

Katgert, P., Mazure, A., den Hartog, R., et al. 1998, A\&AS, 129, 399

Kodama, T., \& Arimoto, N. 1997, A\&A, 320, 41

Lage, C., \& Farrar, G. R. 2015, JCAP, 2, 38

Mansheim, A. S., Lemaux, B. C., Dawson, W. A., et al. 2017a, ApJ, 834, 205

Mansheim, A. S., Lemaux, B. C., Tomczak, A. R., et al. 2017b, MNRAS, 469, L20

Markevitch, M., Gonzalez, A. H., David, L., et al. 2002, ApJL, 567, L27

Maurogordato, S., Cappi, A., Ferrari, C., et al. 2008, A\&A, 481, 593

Maurogordato, S., Proust, D., Beers, T. C., et al. 2000, A\&A, 355, 848

Miller, N. A., \& Owen, F. N. 2003, AJ, 125, 2427

Miyazaki, S., Komiyama, Y., Sekiguchi, M., et al. 2002, PASJ, 54, 833

Moore, B., Katz, N., Lake, G., Dressler, A., \& Oemler, A. 1996, Natur, 379,613

Newman, A. B., Treu, T., Ellis, R. S., et al. 2013a, ApJ, 765, 24

Newman, J. A., Cooper, M. C., Davis, M., et al. 2013b, ApJS, 208, 5

Ng, K. Y., Dawson, W. A., Wittman, D., et al. 2015, MNRAS, 453, 1531

Ouchi, M., Shimasaku, K., Okamura, S., et al. 2004, ApJ, 611, 660

Owers, M. S., Nulsen, P. E. J., Couch, W. J., et al. 2014, ApJ, 780, 163

Owers, M. S., Randall, S. W., Nulsen, P. E. J., et al. 2011, ApJ, 728, 27

Pierre, M., Oukbir, J., Dubreuil, D., et al. 1997, A\&AS, 124, 283

Poggianti, B. M., Bridges, T. J., Komiyama, Y., et al. 2004, ApJ, 601, 197

Randall, S. W., Markevitch, M., Clowe, D., Gonzalez, A. H., \& Bradač, M. 2008, ApJ, 679, 1173

Schlafly, E. F., \& Finkbeiner, D. P. 2011, ApJ, 737, 103

Schlegel, D. J., Finkbeiner, D. P., \& Davis, M. 1998, ApJ, 500, 525

Sehgal, N., Hughes, J. P., Wittman, D., et al. 2008, ApJ, 673, 163

Skillman, S. W., Xu, H., Hallman, E. J., et al. 2013, ApJ, 765, 21

Skrutskie, M. F., Cutri, R. M., Stiening, R., et al. 2006, AJ, 131, 1163

Sobral, D., Stroe, A., Dawson, W. A., et al. 2015, MNRAS, 450, 630

Stroe, A., Sobral, D., Paulino-Afonso, A., et al. 2017, MNRAS, 465, 2916

Stroe, A., Sobral, D., Röttgering, H. J. A., \& van Weeren, R. J. 2014, MNRAS, 438, 1377

Takizawa, M., Nagino, R., \& Matsushita, K. 2010, PASJ, 62, 951

Taylor, M. B. 2005, in ASP Conf. Ser. 347, Astronomical Data Analysis Software and Systems XIV, ed. P. Shopbell, M. Britton, \& R. Ebert (San Francisco, CA: ASP), 29

Valtchanov, I., Murphy, T., Pierre, M., Hunstead, R., \& Lémonon, L. 2002, A\&A, 392, 795

van Weeren, R. J., Andrade-Santos, F., Dawson, W. A., et al. 2017, NatAs, 1,0005

van Weeren, R. J., Bonafede, A., Ebeling, H., et al. 2012a, MNRAS, 425, L36

van Weeren, R. J., Brüggen, M., Röttgering, H. J. A., et al. 2011a, A\&A, 533, A35

van Weeren, R. J., Fogarty, K., Jones, C., et al. 2013, ApJ, 769, 101

van Weeren, R. J., Hoeft, M., Röttgering, H. J. A., et al. 2011b, A\&A, 528, A38

van Weeren, R. J., Röttgering, H. J. A., Bagchi, J., et al. 2009b, A\&A, 506, 1083 van Weeren, R. J., Röttgering, H. J. A., \& Brüggen, M. 2011c, A\&A, 527, A114 van Weeren, R. J., Röttgering, H. J. A., Brüggen, M., \& Cohen, A. 2009a, A\&A, 508, 75

van Weeren, R. J., Röttgering, H. J. A., Brüggen, M., \& Hoeft, M. 2010, Sci, 330,347

van Weeren, R. J., Röttgering, H. J. A., Intema, H. T., et al. 2012b, A\&A, 546, A124

Vazza, F., Brüggen, M., van Weeren, R., et al. 2012, MNRAS, 421, 1868

Vazza, F., Brüggen, M., Wittor, D., et al. 2016, MNRAS, 459, 70

Voges, W., Aschenbach, B., Boller, T., et al. 1999, A\&A, 349, 389

Wittor, D., Vazza, F., \& Brüggen, M. 2017, MNRAS, 464, 4448

Yagi, M., Kashikawa, N., Sekiguchi, M., et al. 2002, AJ, 123, 66

Zabludoff, A. I., Huchra, J. P., \& Geller, M. J. 1990, ApJS, 74, 1

Zhang, Y.-Y., Okabe, N., Finoguenov, A., et al. 2010, ApJ, 711, 1033

Ziparo, F., Braglia, F. G., Pierini, D., et al. 2012, MNRAS, 420, 2480 